\title{
Distinct immune characteristics distinguish hereditary and idiopathic chronic pancreatitis
}

\author{
Bomi Lee, ${ }^{1}$ Julia Z. Adamska, ${ }^{1}$ Hong Namkoong, ${ }^{1}$ Melena D. Bellin, ${ }^{2,3}$ Josh Wilhelm, ${ }^{2}$ Gregory L. Szot, ${ }^{4}$ David M. Louis, ${ }^{5}$ \\ Mark M. Davis, ${ }^{5,6,7}$ Stephen J. Pandol, ${ }^{8}$ and Aida Habtezion ${ }^{1,6}$ \\ 'Division of Gastroenterology and Hepatology, Department of Medicine, Stanford University Medical School, Stanford, California, USA. ${ }^{2 S c h u l z e ~ D i a b e t e s ~ I n s t i t u t e, ~ D e p a r t m e n t ~ o f ~ S u r g e r y, ~ U n i v e r s i t y ~ o f ~}$ \\ Minnesota Medical Center, Minneapolis, Minnesota, USA. ${ }^{3}$ Department of Pediatrics, University of Minnesota Medical Center and Masonic Children's Hospital, Minneapolis, Minnesota, USA. ${ }^{4}$ Division of \\ Transplantation, Department of Surgery, UCSF, San Francisco, California, USA. ${ }^{5}$ Department of Microbiology and Immunology, ${ }^{6}$ Institute for Immunity, Transplantation and Infection, and 'Howard Hughes \\ Medical Institute (HHMI), Stanford University Medical School, Stanford, California, USA. ${ }^{8}$ Department of Medicine, Cedars-Sinai Medical Center, Los Angeles, California, USA.
}

\begin{abstract}
Chronic pancreatitis (CP) is considered an irreversible fibroinflammatory pancreatic disease. Despite numerous animal model studies, questions remain about local immune characteristics in human CP. We profiled pancreatic immune cell characteristics in control organ donors and CP patients including those with hereditary and idiopathic CP undergoing total pancreatectomy with islet autotransplantation. Flow cytometric analysis revealed a significant increase in the frequency of CD68 ${ }^{+}$ macrophages in idiopathic CP. In contrast, hereditary CP samples showed a significant increase in $C D 3^{+} \mathrm{T}$ cell frequency, which prompted us to investigate the T cell receptor $\beta$ (TCR $\beta$ ) repertoire in the CP and control groups. TCR $\beta$ sequencing revealed a significant increase in TCR $\beta$ repertoire diversity and reduced clonality in both CP groups versus controls. Interestingly, we observed differences in V $\beta$-J $\beta$ gene family usage between hereditary and idiopathic CP and a positive correlation of TCR $\beta$ rearrangements with disease severity scores. Immunophenotyping analyses in hereditary and idiopathic CP pancreases indicate differences in innate and adaptive immune responses, which highlights differences in immunopathogenic mechanisms of disease among subtypes of CP. TCR repertoire analysis further suggests a role for specific T cell responses in hereditary versus idiopathic CP pathogenesis, providing insights into immune responses associated with human CP.
\end{abstract}

\section{Introduction}

Chronic pancreatitis (CP) is an inflammatory disease of the pancreas and remains a major source of morbidity in the United States and Europe (1). CP is associated with an irreversible destruction of the pancreas parenchyma and fibrosis accompanied by severe abdominal pain, which leads to poor quality of life $(2,3)$. Alcohol and smoking are established as major etiological factors in adult CP. Still, genetic variants and other idiopathic factors account for up to $20 \%$ of the cases, and genetic variants are especially a common risk factor in pediatric $\mathrm{CP}$ $(4-7)$. Despite its prevalence, cost, and societal impact, there is no active approved therapy or early diagnostic marker for CP. Inflammation is a known hallmark of CP and its pathogenesis, as demonstrated by studies of pancreas immune cell infiltrations in humans $(8-10)$ and experimental models $(11,12)$. Although many animal models have been used to study CP pathogenesis, questions remain regarding the translational accuracy of preclinical studies. Based on experimental model studies, the assumption in the field has been that the immune responses in $\mathrm{CP}$ are uniform or similar regardless of the etiology of CP; however, this remains to be proven in human disease.

Conflict of interest: The authors have declared that no conflict of interest exists. Copyright: ( 2020, American Society for Clinical Investigation.

Submitted: October 7, 2019; Accepted: February 11, 2020; Published: April 20, 2020

Reference information: J Clin Invest. 2020;130(5):2705-2711.

https://doi.org/10.1172/JCl134066.
Human studies are hampered by lack of tissue access, and as a result many studies rely on analysis of peripheral blood mononuclear cells, which might not necessarily reflect local immune responses. To address this gap in the field, we collected pancreas tissues from CP patients undergoing total pancreatectomy with islet autotransplantation (TPIAT) (13-15) and control pancreas tissues subjected to the same islet isolation from deceased organ donors with no known history of pancreatic disease. Unexpectedly, we found that different types of CP had distinct immune cell composition and $\mathrm{T}$ cell receptor $\beta$ (TCR $\beta$ ) repertoires, such as TCR $\beta$ gene usage and rearrangements. Interestingly, TCR $\beta$ rearrangement counts in $\mathrm{CP}$ patients positively correlated with disease severity scores. These findings suggest that there are differences in the immune mechanisms underlying hereditary versus idiopathic $\mathrm{CP}$ and their association with $\mathrm{CP}$ disease pathogenesis.

\section{Results and Discussion}

Pancreas tissues from CP patients $(n=40)$ undergoing TPIAT and from non-CP deceased organ donors $(n=9)$ that underwent similar islet extraction were collected consecutively from the University of Minnesota and UCSF, respectively. Characteristics were comparable in the 2 groups, except for age, weight, and BMI (Supplemental Table 1; supplemental material available online with this article; https://doi.org/10.1172/JCI134066DS1). Twenty-seven hereditary and 13 idiopathic CP patients without known history of diabetes were included in the study. Significant differences were observed in age, sex, BMI, gross fibrosis severity 
A

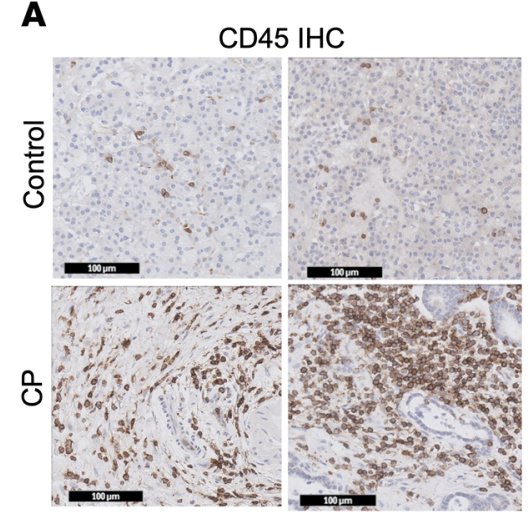

B

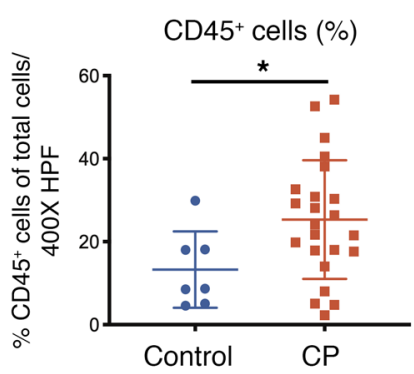

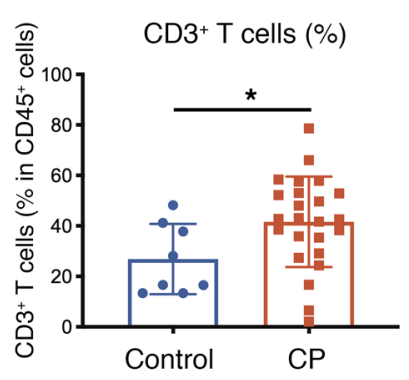

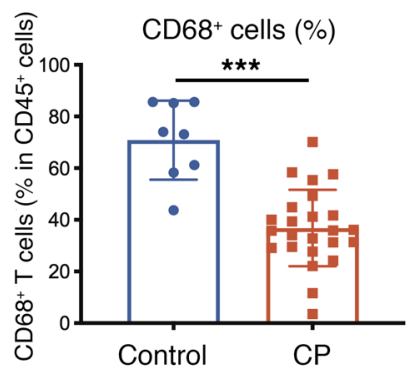

C

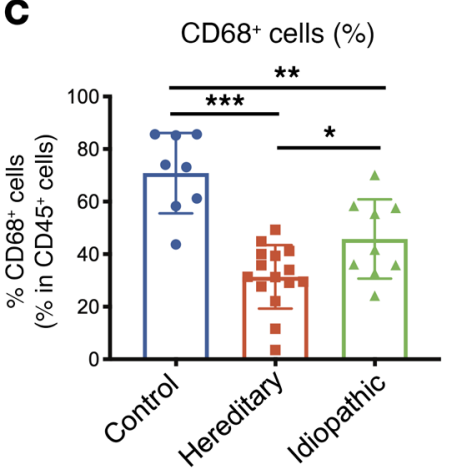

D

Luminex data (hereditary vs. idiopathic)

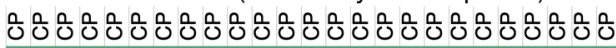

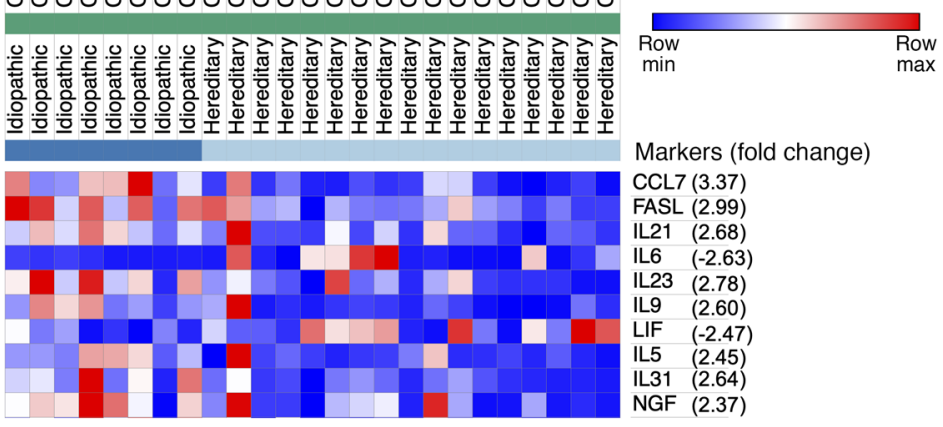

$\mathrm{CD}^{+} 8^{+} \mathrm{CD} 11 \mathrm{c}^{+}$cells $(\%)$
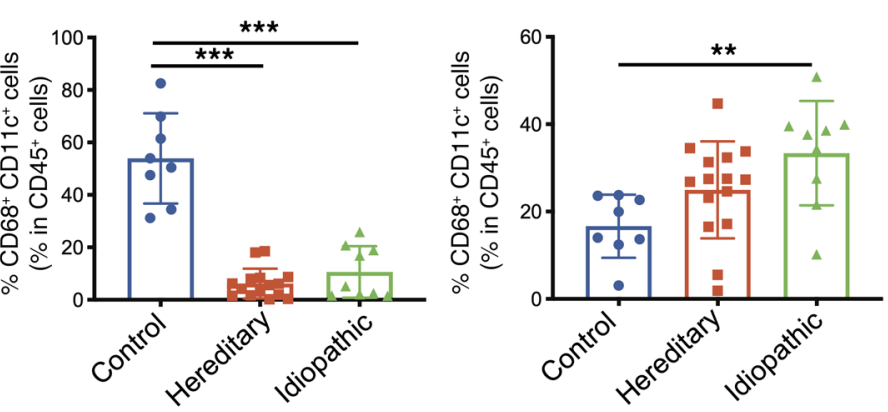

E

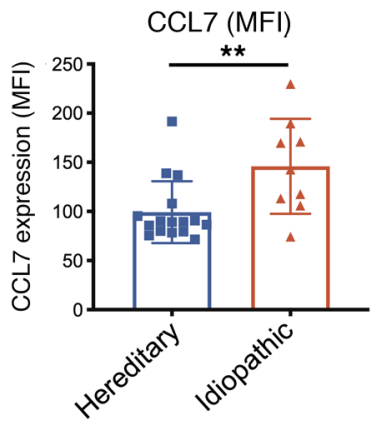

Figure 1. CD68+ macrophages are predominant in idiopathic CP compared with hereditary CP. (A) Immunohistochemistry staining using the pan-leukocyte marker CD45 (original magnification, $\times 400$ ). Scale bars: $100 \mu \mathrm{m}$. The percentage of CD45+ cells among total pancreatic cells is presented as a dot plot. Mean \pm SD; unpaired 2-tailed $t$ test. (B) The frequency of $\mathrm{CD}^{+}$T cells and CD68 cells in live CD45+ cells from control $(n=8)$ and CP $(n=24)$ samples. Mean \pm SD; unpaired 2-tailed $t$ test was used. (C) Graphs show frequencies of CD68+ cells and their subsets in live CD45 cells from control $(n=8)$, hereditary CP $(n=15)$, and idiopathic CP $(n=9)$ tissues. Mean \pm SD; 1-way ANOVA with Tukey's multiple-comparisons test. (D) Heatmap represents expression levels of analytes with MFI values by the human 62 multiplex Luminex assay ( $t$ test, $P<0.05$, FDR $<0.25$ ). Fold change in the average expression of each analyte in idiopathic versus hereditary CP. (E) Comparison of MFI values of the most differentially regulated chemokine (CCL7) between hereditary $(n=17)$ and idiopathic $(n=8)$ CP. Mean \pm SD; unpaired 2-tailed $t$ test was used. ${ }^{*} P<0.05,{ }^{* *} P<0.01,{ }^{* *} P<0.001$. HPF, high-power field.

score, and cigarette smoking history between the $2 \mathrm{CP}$ groups (Supplemental Table 2). Human pancreas tissues were used for the different immune analyses, including flow cytometry, Luminex assay, and TCR $\beta$ sequencing (TCR $\beta$-Seq) (Supplemental Figure 1, A and B).

As expected, deposits of $\mathrm{CD}_{4} 5^{+}$(pan-leukocyte marker-positive) cells were significantly larger in CP compared with control pancreas tissues (Figure 1A). Given the increased leukocyte levels in $\mathrm{CP}$, we sought to profile the immune subsets infiltrating of CP compared with control pancreas using flow cytometry. Innate and adap- tive immune subset characterization included the identification of macrophage subsets, T cell subsets, mast cells, NK cells, and NKT cells (Supplemental Figure 2). Interestingly, the frequency of $\mathrm{CD} 3^{+}$ $\mathrm{T}$ cells was significantly increased in CP compared with control tissues. Even though the absolute count of $\mathrm{CD} 68^{+}$macrophages was increased in $\mathrm{CP}$, their proportion within live $\mathrm{CD} 45^{+}$leukocytes was significantly reduced in CP compared with control tissues (Figure 1B), potentially due to the increased proportion of T cells. Among $\mathrm{CD} 68^{+}$cells, the percentage of $\mathrm{CD} 68^{+} \mathrm{CD} 11 \mathrm{c}^{-}$cells significantly increased in $\mathrm{CP}$, while the proportion of $\mathrm{CD} 68^{+} \mathrm{CD} 11 \mathrm{c}^{+}$cells was 
A
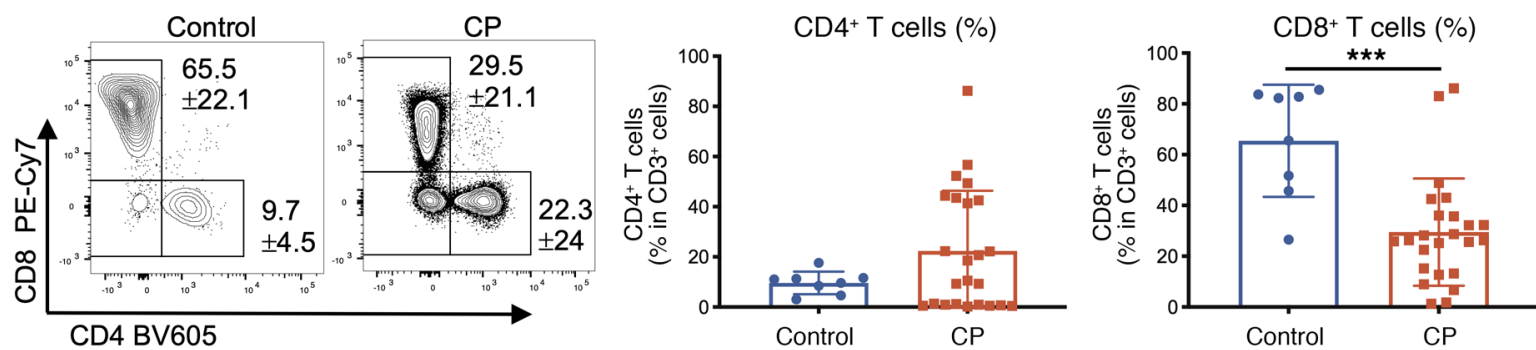

B
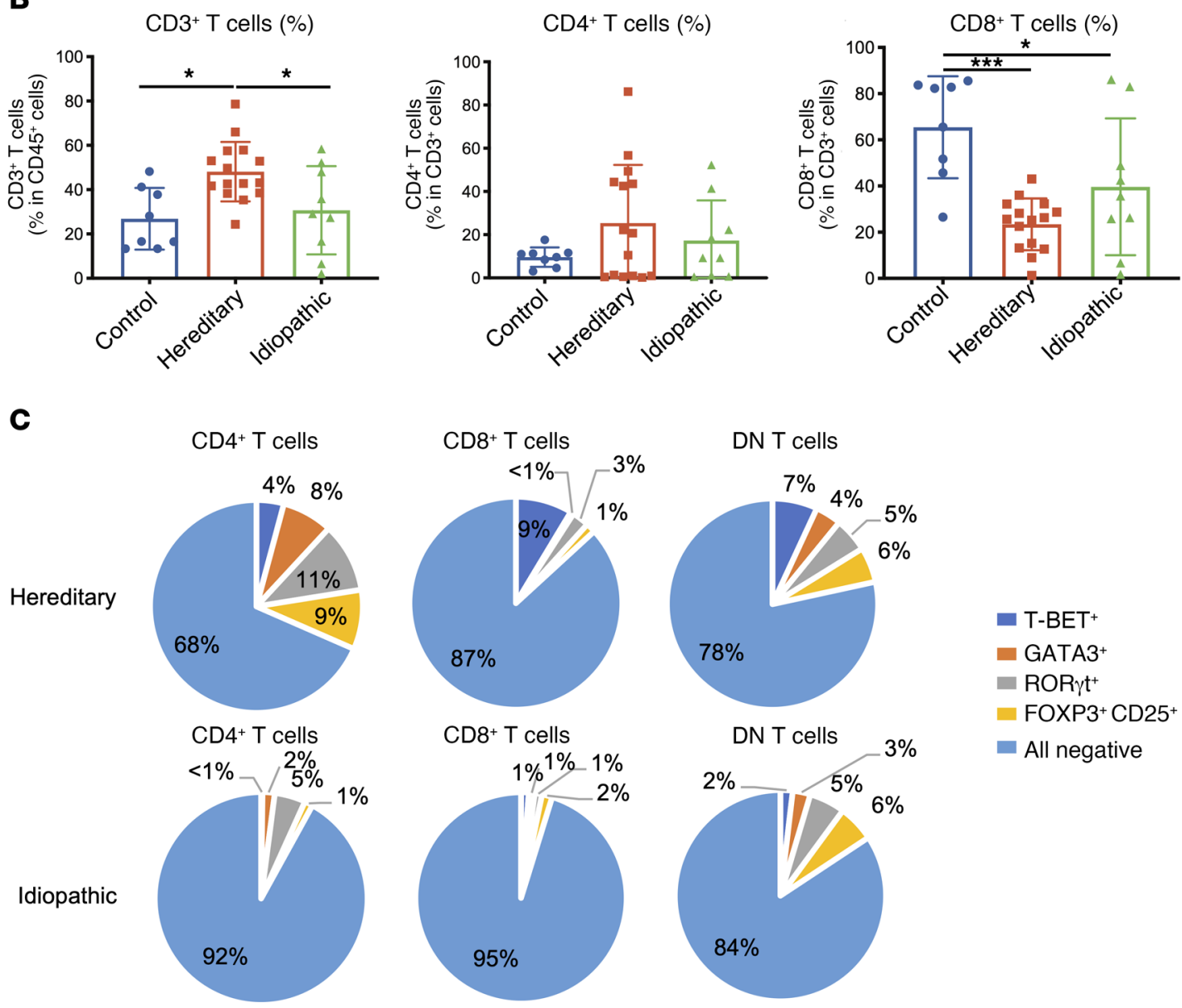

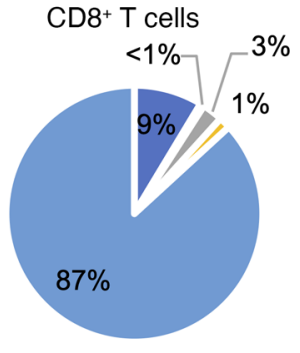

CD8 ${ }^{+} \mathrm{T}$ cells

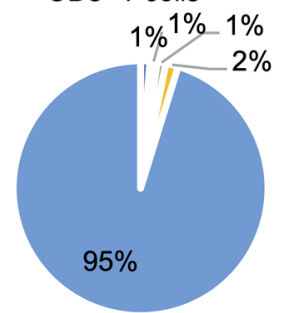

DN T cells

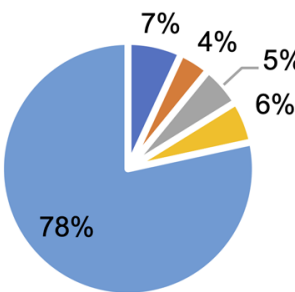

DN T cells

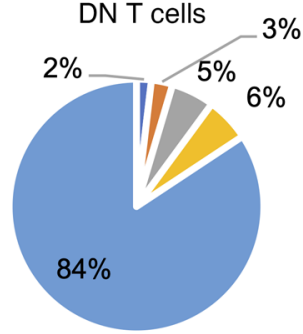

- T-BET+

- GATA3 ${ }^{+}$

- ROR $\mathrm{H}^{+}$

FOXP3 ${ }^{+}$CD25+

All negative

Figure 2. $\mathrm{CD3}^{+} \mathbf{T}$ cells are more frequent in hereditary $\mathbf{C P}$ compared with idiopathic $\mathbf{C P}$. (A) Representative plots of flow cytometry analyses of $\mathrm{CD3^{+ }}{ }^{\mathrm{T}}$ cells based on CD4 and CD8 expression in control $(n=8)$ and $C P(n=24)$ samples (mean \pm SD). Graphs show frequency of CD4+ or CD8 ${ }^{+}$T cells in control and CP samples (mean $\pm \mathrm{SD}$; unpaired 2-tailed $t$ test). (B) Frequencies of total $\mathrm{CD}^{+}, \mathrm{CD}^{+}$, and $\mathrm{CD} 8^{+}$T cells among live $\mathrm{CD} 45^{+}$cells from control $(n=8)$, hereditary CP $(n=15)$, and idiopathic CP $(n=9)$ tissues. Mean \pm SD; 1-way ANOVA with Tukey's multiple-comparisons test. (C) Pie charts represent the average frequencies of T-bet ${ }^{+}, \mathrm{CATA}^{+}, \mathrm{ROR} \gamma \mathrm{t}^{+}$, and FOXP3/CD25+ $\mathrm{T}$ cell subsets in $\mathrm{CD}^{+}, \mathrm{CD} 8^{+}$, or double-negative (DN) T cells from hereditary $(n=15)$ and idiopathic $(n=9)$ CP tissues. ${ }^{*} P<0.05,{ }^{* * *} P<0.001$.

significantly reduced in CP compared with control pancreases (Supplemental Figure $3 \mathrm{~A})$. The $\mathrm{CD} 68^{+} \mathrm{CD} 11 \mathrm{c}^{+}$population was enriched for cells expressing high levels of HLA-DR and CD11b, likely representing homeostatic functions such as antigen presentation and phagocytosis. Inversely, $\mathrm{CP}$ tissues appeared to have expanded CD68 ${ }^{+} \mathrm{CD} 11 \mathrm{c}^{-}$cells that were negative or low for HLA-DR and CD11b expression (Supplemental Figure 3B).

Among the CP cases undergoing TPIAT in this study, a substantial proportion had hereditary/genetic mutations, such as in
PRSS1, SPINK1, CFTR, and CTRC, and the second most common etiology was classified as idiopathic $\mathrm{CP}$ without particular causes identified. When we compared these 2 cohorts, the frequency of $\mathrm{CD} 68^{+}$cells was significantly higher in idiopathic as compared with hereditary $\mathrm{CP}$ (Figure 1C). Among the $\mathrm{CD} 68^{+}$cell populations, the proportion of $\mathrm{CD} 68^{+} \mathrm{CD} 11 \mathrm{c}^{-}$cells was significantly increased in idiopathic $\mathrm{CP}$ as compared with control, while $\mathrm{CD} 68^{+} \mathrm{CD} 11 \mathrm{c}^{+}$cell frequency was significantly lower in the both $\mathrm{CP}$ groups compared with controls (Figure 1C). Our group previously reported that anti- 
A
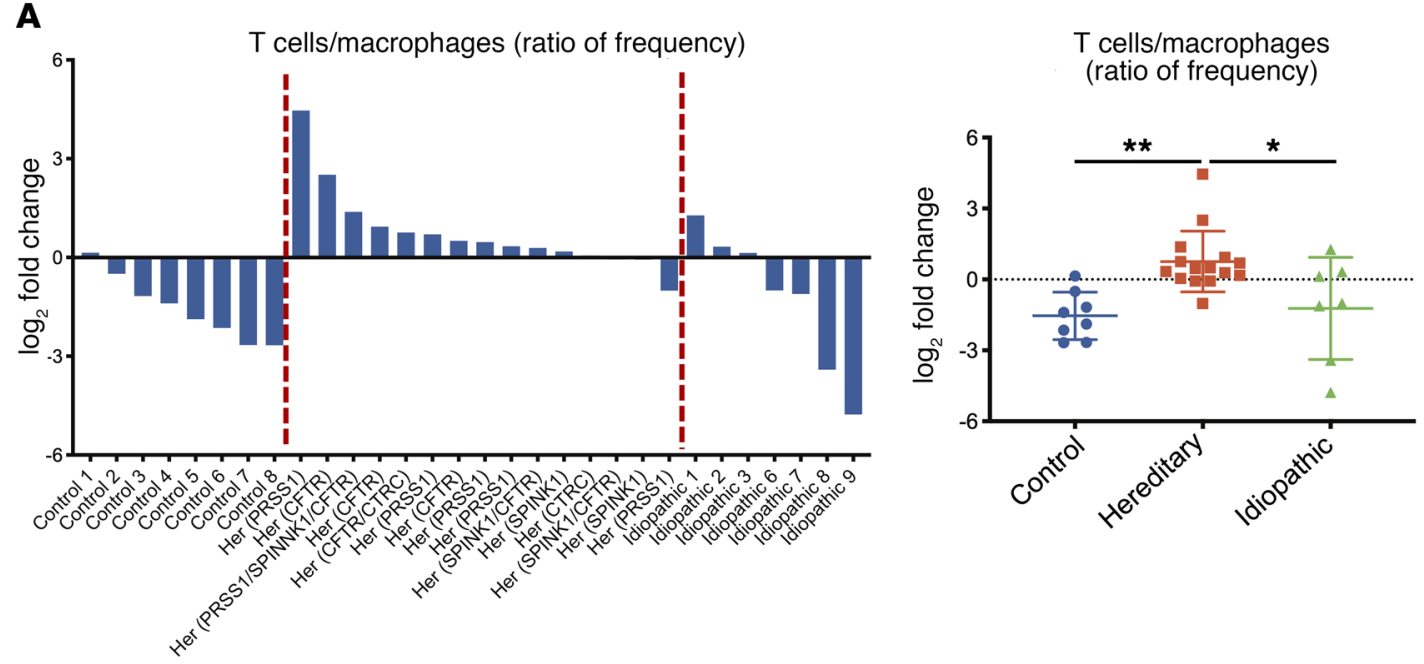

B

Top 100 most frequent clones

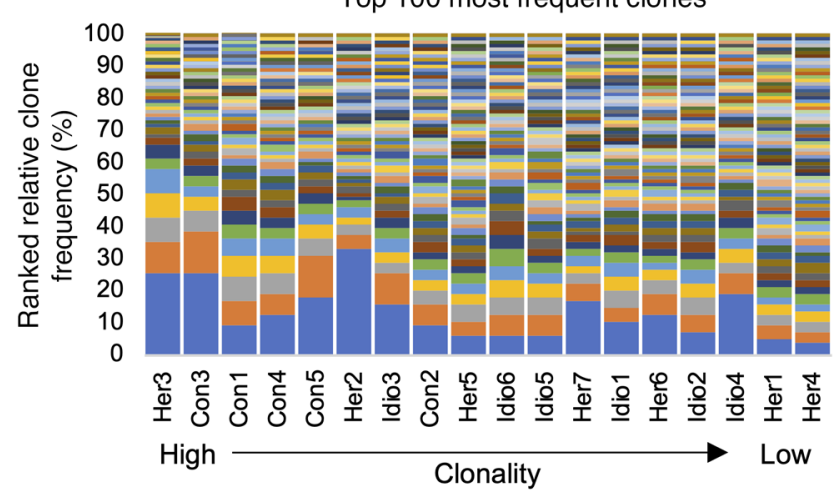

C
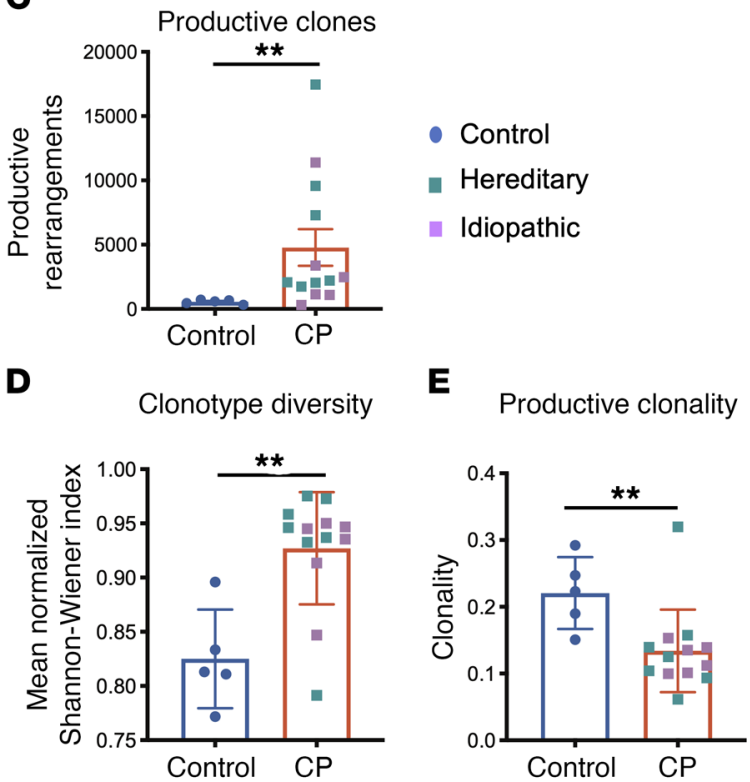

Figure 3. TCR $\beta$ repertoire of control and CP pancreatic T cells. (A) Waterfall and dot plots show the ratio of CD $3^{+} \mathrm{T}$ cell to $C D 68^{+}$macrophage frequency in control, hereditary CP, and idiopathic CP tissues. Identified gene mutations are indicated in an individual hereditary CP patient. Mean \pm SD; 1 -way ANOVA with Tukey's multiple-comparisons test. Her, hereditary. (B) One hundred most frequent rearrangements in each sample ranked from bottom (most frequent clone) to top (100th most frequent clone), and samples are listed by their clonality order from left to right. Number of productive rearrangements (C), TCR clonotype diversity (mean normalized Shannon-Wiener diversity index) (D), and productive clonality are shown (E). (C-E) Comparison between control $(n=5)$ and CP $(n=13)$. Nonparametric Mann-Whitney $U$ test. ${ }^{*} P<0.05,{ }^{* *} P<0.01$.

inflammatory (M2) macrophages are predominant in mouse CP and surgically resected pancreas specimens from human CP (11). Consistent with previous results, M2 macrophages were predominant in the $\mathrm{CD} 68^{+} \mathrm{CD} 11 \mathrm{c}^{-}$population of idiopathic and hereditary $\mathrm{CP}$, whereas no significant difference was observed between frequencies of $\mathrm{M} 1$ and $\mathrm{M} 2$ macrophages in the $\mathrm{CD} 68^{+} \mathrm{CD} 11 \mathrm{c}^{+}$population of both CP groups (Supplemental Figure 4, A and B). Thus, even within the $2 \mathrm{CP}$ subtypes, there were notable differences in immune cell composition, with an expansion of $\mathrm{CD} 68^{+}$cells in idiopathic $\mathrm{CP}$ compared with hereditary CP. In addition, there was prominent differential expression of cytokines and chemokines between the hereditary and idiopathic CP groups, as indicated by Luminex assay (Figure 1D). Consistent with the increased portion of $\mathrm{CD} 68^{+}$macrophages in idiopathic $\mathrm{CP}$ compared with hered- itary CP, CCL7 (monocyte chemotactic protein 3), was the most significantly increased analyte in idiopathic versus hereditary $\mathrm{CP}$ (Figure 1E). Other significant differences included an increased ratio of M2 (IL-4, IL-13) to M1 (TNFA) cytokine expression in idiopathic versus hereditary CP (Supplemental Figure 4C); and higher innate (IL-21, IL-23) and Th2 (IL-5, IL-9, IL-31) cytokine levels in idiopathic CP, whereas IL-6 and LIF levels were higher in hereditary CP (Figure 1D). Taken together with the macrophage profiles above, these cytokine profiles suggest that idiopathic $\mathrm{CP}$ is locally enriched in innate immune cells, i.e., macrophages, compared with hereditary CP.

Next, we compared the composition of the $\mathrm{T}$ cell populations. Among $\mathrm{CD} 3^{+} \mathrm{T}$ cells, the percentage of $\mathrm{CD} 4^{+} \mathrm{T}$ cells was increased in $\mathrm{CP}$, while that of $\mathrm{CD} 8^{+} \mathrm{T}$ cells was significantly diminished in 
A
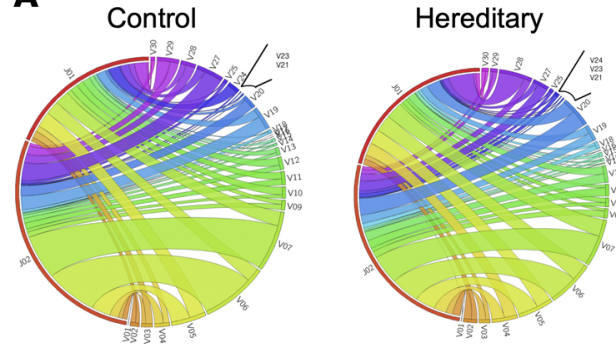

B

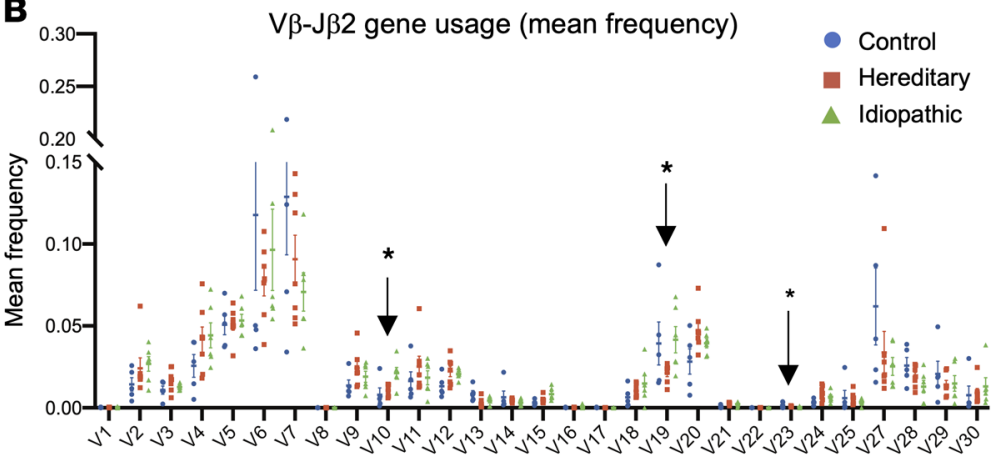

D

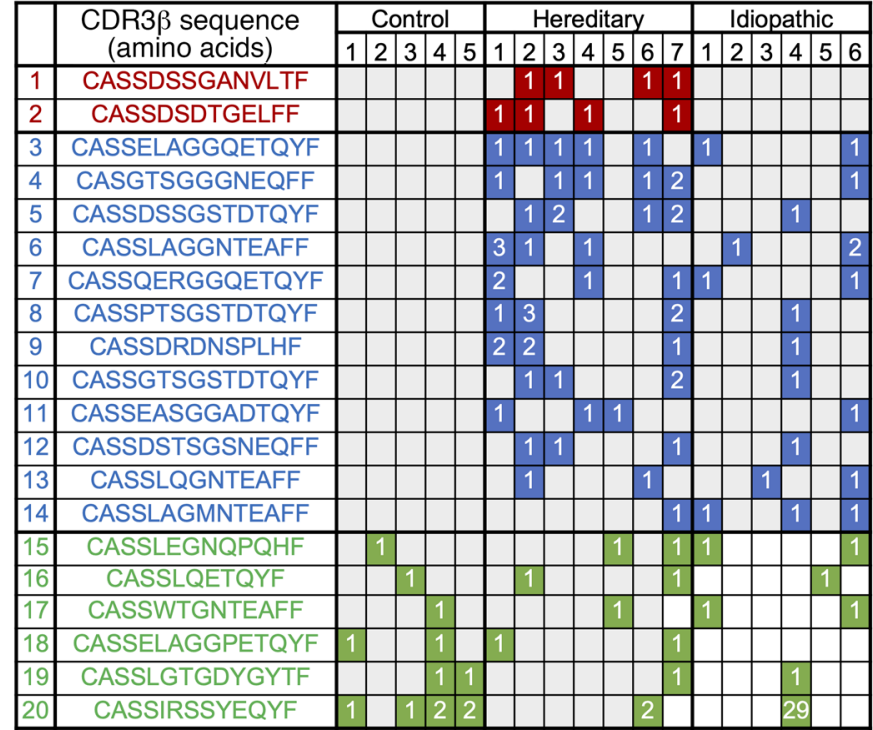

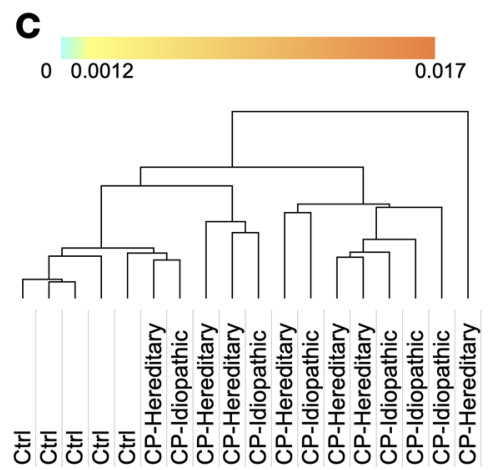

TRBV9-1 \& J2-2 TRBV7-2 \& J2-7 TRBV6-6 \& J2-7 TRBV6-1 \& J1-6 TRBV5-4 \& J2-5 TRBV4-2 \& J2-3 TRBV28-1 \& J1-5 TRBV18-1 \& J1-4 TRBV18-1 \& J1-1

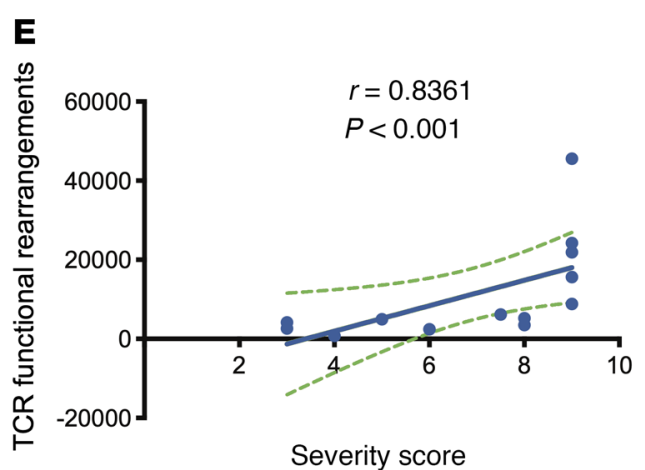

Figure 4. Differences in TCR $\beta$ repertoire between hereditary and idiopathic CP pancreatic T cells. (A) Circos plots indicate frequencies of V $\beta$-J $\beta$ productive gene usage in control $(n=5)$, hereditary CP $(n=7)$, and idiopathic CP $(n=6)$ cells. The width of the V $\beta$-J $\beta$ pair band is proportional to the frequency in each group. (B) Comparison of V $\beta$-J $\beta 2$ gene family usage among groups, shown as mean frequencies. Mean $\pm \mathrm{SEM}$; 1 -way ANOVA with Kruskal-Wallis test, comparison between hereditary and idiopathic CP; ${ }^{*} P<0.05$. (C) Heatmap representing frequencies of $V \beta$-J $\beta$ gene pairs that are significantly different (significance analysis of microarray $t$ test, 90th percentile FDR $=0$ ) between control and $C P$ cells. (D) Full-length CDR3 amino acid sequences shared among at least 4 subjects. Numbers in squares represent the count of unique clonotypes in a subject's repertoire, with the CDR3 $\beta$ sequence indicated. (E) Correlation of the number of functional TCR rearrangements with CP disease severity score $(n=13$, nonparametric Spearman's correlation $r=0.8361, P<0.001)$.

CP compared with control tissues (Figure 2A and Supplemental Figure $5 \mathrm{~A}$ ). Unlike the macrophage findings above, there was a significantly higher proportion of $\mathrm{CD}^{+} \mathrm{T}$ cells in hereditary $\mathrm{CP}$ compared with control or idiopathic CP cells (Figure 2B). Upon stratification of the $\mathrm{T}$ cells, there was a trend toward increased proportions of $\mathrm{CD}^{+} \mathrm{T}$ cells in both $\mathrm{CP}$ groups versus controls, whereas $\mathrm{CD}^{+} \mathrm{T}$ cell frequency was significantly reduced in both $\mathrm{CP}$ groups compared with controls (Figure $2 \mathrm{~B}$ and Supplemental
Figure 5B). Interestingly, $\mathrm{CD}^{+} \mathrm{T}$ cell subpopulations including T-bet $^{+}$(Th1), GATA3 ${ }^{+}$(Th2), ROR $\gamma \mathrm{t}^{+}$(Th17/22), and CD25 ${ }^{+} \mathrm{FOXP3}^{+}$ (Treg) were expanded to a greater extent in hereditary than idiopathic $\mathrm{CP}$, as indicated by the average frequencies of different $\mathrm{T}$ cell subpopulations (Figure 2C); this suggests that functionally active $\mathrm{CD}^{+}$helper $\mathrm{T}$ cell subsets may play critical roles in the pathogenesis of hereditary CP. Typically, CP is characterized by a fibrotic condition with injury-driven inflammatory responses (16). 
Innate and adaptive immune cells contribute to pathologic fibrosis in different diseases $(17,18)$. We examined fibrosis of pancreatic tissues by trichrome staining. Both CP tissues showed a significantly higher proportion of fibrotic tissue area compared with controls (Supplemental Figure 6, A and B). Although there was a trend toward higher fibrosis in hereditary CP, this was not statistically significant between the 2 CP groups (Supplemental Figure 6C).

As revealed by flow cytometry analyses with pancreatic immune cells, the ratio of $\mathrm{CD}^{+} \mathrm{T}$ cell frequency to $\mathrm{CD} 68^{+}$macrophage frequency in the pancreas was significantly higher in hereditary versus idiopathic CP (Figure 3A). Hereditary CP had a distinct immune cell distribution, with a greater $\mathrm{T}$ cell proportion, whereas idiopathic CP had expanded CD $68^{+}$macrophages, suggesting unique immune mechanisms underlying the different etiologies of the CP groups. TCR clonality and diversity have been found to affect a wide variety of disease conditions, including malignancy and autoimmune disorders (19-21). Given the differences in immune profiling among CP pancreases, we hypothesized there might be alterations of the TCR repertoire in pancreas-infiltrating $\mathrm{T}$ cells. To investigate this, we isolated genomic DNA (gDNA) from pancreas tissues of control donors and $\mathrm{CP}$ patients. The TCR $\beta$ repertoire was examined by sequencing the third complementarity-determining region 3 (CDR3) loop of TCR $\beta$ and adjacent regions, which is typically the contact region for engaging antigenic peptides (Supplemental Table 3 and Figure $3 \mathrm{~B}$ ). Numbers of total $\mathrm{T}$ cells and unique rearrangements were significantly increased in the CP groups, especially in the hereditary CP compared with the control group (Figure $3 \mathrm{C}$ and Supplemental Figure 7A). The TCR template diversity index was also significantly higher in both CP groups (Figure 3D and Supplemental Figure 7B, left). However, the productive clonality of CP groups, especially in idiopathic $\mathrm{CP}$, was significantly lower than in the control group (Figure $3 \mathrm{E}$ and Supplemental Figure $7 \mathrm{~B}$ right). These results indicate that the increased number of $\mathrm{T}$ cells in CP was due not to the clonal expansion of infiltrating $\mathrm{T}$ cells, but rather to the increased variety of $\mathrm{T}$ cell clonotypes. Next, we compared CDR3 $\beta$ length distribution as an indicator of TCR repertoire change and $\mathrm{T}$ cell response to antigens $(22,23)$. CDR3 $\beta$ length in both CP groups tended to be shorter than in the control group, although the mean lengths were not significantly different (Supplemental Figure 7C).

Next, we looked at the pattern of TCR $\beta$ V-J paired gene family usage among CP groups and identified shared V-J gene combinations among the groups. The pattern of V-J gene family usage was comparable across groups, and the 3 most dominant $V \beta$ gene families in all groups were TRBV7, -6 , and -5 (Figure $4 \mathrm{~A}$ ). The mean frequency of the $\mathrm{V} \beta$ gene paired with $\mathrm{J} \beta 2$ showed $\mathrm{V} \beta$ gene families including TRBV10, -19, and -23 differentially used between hereditary and idiopathic CP (Figure 4B), whereas comparison of the V $\beta$-J $\beta 1$ gene family showed TRBV5 as a differentially used gene family between the $2 \mathrm{CP}$ groups (Supplemental Figure 7D). We next sought to further analyze $V \beta-J \beta$ gene combinations in the CP groups compared with control and found 9 significantly differentially used V $\beta-J \beta$ gene combinations (Figure $4 C$ ). Further, we examined inter-repertoire homology among patients by identifying shared CDR3 $\beta$ s representing identical amino acid composition and length (Figure 4D). We found 20 identical
CDR3 $\beta$ s shared by at least 4 subjects, with some CDR3 $\beta$ s shared only among hereditary CP patients, or only CP patients, and others shared by controls and CP patients. It is noteworthy that the number of productive, functional TCR rearrangements positively correlated with disease severity score in CP (Figure 4E). As the disease severity score is determined with consideration of multiple factors, such as calcification, cysts, parenchyma color, blood content, fat content, fibrosis, and ductal destruction, a positive correlation between disease severity score and functional TCR clonotype counts indicates the importance of the relationship between the TCR $\beta$ repertoire and CP disease progression. Overall, the TCR $\beta$-Seq data revealed an imprint of distinct antigenic repertoires in the $\mathrm{CP}$ groups compared with control, as well as distinct mechanisms underlying disease-associated pancreasinfiltrating $\mathrm{T}$ cells in different subtypes of CP.

Here we uncovered distinct local immune characteristics in different subtypes of human CP, hereditary and idiopathic. Our study did not include CP with alcohol use as its etiology, due to the fact that an insufficient number of these patients undergo TPIAT (24). Remarkably, increased T cell frequency was found in hereditary versus idiopathic $\mathrm{CP}$, whereas the proportion of macrophages was greater in idiopathic compared with hereditary CP. This finding indicates that distinct immune subpopulation-mediated mechanisms may underlie the pathogenesis of CP of different etiologies. Since we could only access and analyze the tissue at the time of TPIAT, it is possible that differences in disease duration and extent of histological changes may have influenced distinct immune responses in the different CP groups. Although we found no significant difference in tissue fibrosis between the $2 \mathrm{CP}$ groups that we used for our immune analysis, it would be worthwhile to examine immune responses over time during CP progression if repeat tissue biopsies become safe and available in CP patients in the future. Our data also bring to light the translational aspect of animal model studies. Most if not all CP models are not genetically driven, and interestingly, the immune profile of these models resembles that of idiopathic CP. It will be interesting in the future to compare the immune characteristics of genetically driven models with our findings in hereditary CP. Given that the average disease severity score (pre-TPIAT clinical measurements) was significantly higher in hereditary CP compared with idiopathic CP and hereditary $\mathrm{CP}$ is associated with a substantially increased risk of pancreatic cancer (6), our results further support potential roles of the T cell-driven immune landscape in CP pathogenesis as well as its progression to malignancy.

To our knowledge, this is the first report of high-throughput TCR $\beta$-Seq with pancreatic T cells from CP patients. Our results unveil the TCR signatures of pancreas-infiltrating $\mathrm{T}$ cells in hereditary and idiopathic CP by using the gDNA isolation method, which involves minimal in vitro experimental manipulation. Our TCR $\beta$-Seq data showed increased T cell infiltrates in CP with increased functional TCR rearrangements and clonotype diversity, suggesting the presence of multiple antigenic or polyclonal $\mathrm{T}$ cell infiltrates in CP; this might be a unique inflammatory feature of CP due to exposure to a variety of insults over disease progression. We also identified CDR3 $\beta$ motifs uniquely shared among hereditary CP or both CP groups, indicating that locally infiltrating $\mathrm{T}$ cells respond to disease-specific antigen targets, 
although further analyses will be necessary to test this notion. A positive correlation between TCR rearrangement counts and disease severity scores found in this study suggests that the TCR repertoire might serve as a prognostic predictor for $\mathrm{CP}$ progression and severity. Since the higher severity score is associated with a decline in islet yield and lower insulin independence rate in CP patients who received TPIAT (25), the number of TCR rearrangements might be an additional predictor and/or pathologic indicator of poor outcome in islet transplantation.

Overall, our studies show distinct immune characteristics in the pancreas of hereditary and idiopathic CP patients, highlighting potential roles of identified immune subpopulations as key regulators of CP pathogenesis. Further, in-depth single-cell-level analysis with antigen/epitope screening is likely to advance our understanding of disease pathogenesis mechanisms for CP syndrome with different etiologies. Such information should contribute to developing the cellular and animal models needed to enhance our understanding of how specific immune responses drive the pathogenesis of these subtypes of $\mathrm{CP}$ and developing $\mathrm{CP}$ subtype-specific therapies.

\section{Methods}

Methods are described in detail in Supplemental Methods.

TCR $\beta$-Seq data. TCR $\beta$-Seq data have been deposited in the Adaptive Biotechnologies immuneACCESS database (https://doi. org/10.21417/BL2020JCI).
Study approval. For the use of human samples in research, the protocol was reviewed and approved by the University of Minnesota and Stanford University Institutional Review Board. Prior to inclusion in the study, written informed consent or parental consent and patient assent, as age appropriate, was received from patient-donors.

\section{Author contributions}

$\mathrm{BL}$ and $\mathrm{AH}$ designed the experiments and wrote the manuscript. $\mathrm{AH}$ provided overall guidance and supervision. $\mathrm{AH}, \mathrm{SJP}, \mathrm{MDB}$, and JW arranged collaborations and/or provided CP patient tissues. GLS provided control pancreatic tissues. BL performed all experiments and analyzed data. HN contributed to flow cytometry panel design and data analysis. JZA and DML contributed to TCR $\beta$-Seq data analysis. MMD, SJP, MDB, and JW reviewed the manuscript and participated in the interpretation of data.

\section{Acknowledgments}

This study was supported in part by NIH grant DK105263 (to AH), a National Pancreas Foundation (NPF) 2019 Research Grant (BL), and the HHMI (MMD, DML). We thank our collaborators, patients, and donors for providing precious human pancreas tissues and the Habtezion laboratory members for their helpful comments.

Address correspondence to: Aida Habtezion, Alway Building, M211, 300 Pasteur Drive, Stanford, California 94305-5187, USA. Phone: 650.725.3362; Email: aidah@stanford.edu.
1. Muniraj T, Aslanian HR, Farrell J, Jamidar PA. Chronic pancreatitis, a comprehensive review and update. Part I: epidemiology, etiology, risk factors, genetics, pathophysiology, and clinical features. Dis Mon. 2014;60(12):530-550.

2. Wilcox CM, et al. Chronic pancreatitis pain pattern and severity are independent of abdominal imaging findings. Clin Gastroenterol Hepatol. 2015;13(3):552-560; quiz e28.

3. Mullady DK, et al. Type of pain, pain-associated complications, quality of life, disability and resource utilisation in chronic pancreatitis: a prospective cohort study. Gut. 2011;60(1):77-84.

4. Whitcomb DC. Genetic aspects of pancreatitis. Annu Rev Med. 2010;61:413-424.

5. Bruno MJ. Chronic pancreatitis. Gastrointest Endosc Clin N Am. 2005;15(1):55-62.

6. French JJ, Charnley RM. Expandable metal stents in chronic pancreatitis. HPB (Oxford). 2003;5(1):58-61.

7. Kumar S, et al. Risk factors associated with pediatric acute recurrent and chronic pancreatitis: lessons from INSPPIRE. JAMA Pediatr. 2016;170(6):562-569.

8. Schmitz-Winnenthal H, et al. Chronic pancreatitis is associated with disease-specific regulatory T-cell responses. Gastroenterology. 2010;138(3):1178-1188.

9. Grundsten M, Liu GZ, Permert J, Hjelmstrom $\mathrm{P}$, Tsai JA. Increased central memory $\mathrm{T}$ cells in patients with chronic pancreatitis. Pancreatology. 2005;5(2-3):177-182.

10. Jupp J, Mansour S, Johnson CD, Sanderson J, Fine $\mathrm{D}$, Gadola S. T-cell populations in chronic pancreatitis. Pancreatology. 2015;15(4):311-312.

11. Xue J, et al. Alternatively activated macrophages promote pancreatic fibrosis in chronic pancreatitis. Nat Commun. 2015;6:7158.

12. Xue J, et al. Aryl hydrocarbon receptor ligands in cigarette smoke induce production of interleukin-22 to promote pancreatic fibrosis in models of chronic pancreatitis. Gastroenterology. 2016;151(6):1206-1217.

13. Sutherland DE, Matas AJ, Najarian JS. Pancreatic islet cell transplantation. Surg Clin North Am. 1978;58(2):365-382.

14. Sutherland DE, et al. Total pancreatectomy and islet autotransplantation for chronic pancreatitis. JAm Coll Surg. 2012;214(4): 409-424; discussion 424.

15. Bellin MD. A role for total pancreatectomy and islet autotransplant in the treatment of chronic pancreatitis. Am J Gastroenterol. 2018;113(3):324-326.

16. Kleeff J, et al. Chronic pancreatitis. Nat Rev Dis Primers. 2017;3:17060.

17. Wangoo A, et al. Contribution of Th1 and Th2 cells to protection and pathology in experimental models of granulomatous lung disease. J Immunol. 2001;166(5):3432-3439.

18. Wick G, Backovic A, Rabensteiner E, Plank N,
Schwentner C, Sgonc R. The immunology of fibrosis: innate and adaptive responses. Trend Immunol. 2010;31(3):110-119.

19. Davis MM, Bjorkman PJ. T-cell antigen receptor genes and T-cell recognition. Nature. 1988;334(6181):395-402.

20. Grupp SA, et al. Adoptive transfer of autologous T cells improves T-cell repertoire diversity and long-term B-cell function in pediatric patients with neuroblastoma. Clin Cancer Res. 2012;18(24):6732-6741.

21. Klarenbeek PL, et al. Inflamed target tissue provides a specific niche for highly expanded T-cell clones in early human autoimmune disease. Ann Rheum Dis. 2012;71(6):1088-1093.

22. Fields BA, et al. Crystal structure of a T-cell receptor beta-chain complexed with a superantigen. Nature. 1996;384(6605):188-192.

23. Gomez-Tourino I, Kamra Y, Baptista R, Lorenc A, Peakman M. T cell receptor $\beta$-chains display abnormal shortening and repertoire sharing in type 1 diabetes. Nat Commun. 2017;8(1):1792.

24. Chinnakotla S, et al. Factors predicting outcomes after a total pancreatectomy and islet autotransplantation lessons learned from over 500 cases. Ann Surg. 2015;262(4):610-622.

25. Chinnakotla S, et al. Long-term outcomes of total pancreatectomy and islet auto transplantation for hereditary/genetic pancreatitis. J Am Coll Surg. 2014;218(4):530-543. 